\title{
Preoperative soluble cluster of differentiation 40 ligand level is associated with outcome of pulmonary endarterectomy
}

\author{
Ayako Shigeta, MD, PhD, ${ }^{\mathrm{a}}$ Nobuhiro Tanabe, MD, PhD, FCCP, ${ }^{\mathrm{a}, \mathrm{b}}$ Akira Naito, MD, PhD, ${ }^{\mathrm{a}}$ \\ Hajime Yokota, MD, PhD, ${ }^{\mathrm{c}}$ Fumiaki Kato, MD, PhD, ${ }^{\mathrm{a}}$ Takayuki Jujo-Sanada, MD, PhD, ${ }^{a}$ \\ Seiichiro Sakao, MD, PhD, ${ }^{a}$ Keiichi Ishida, MD, PhD, ${ }^{d, e}$ Masahisa Masuda, MD, $\mathrm{PhD},{ }^{\mathrm{d}, \mathrm{e}}$ and \\ Koichiro Tatsumi, MD, $\mathrm{PhD}^{\mathrm{a}}$
}

\section{ABSTRACT}

Objective: Soluble $\mathrm{CD} 4 \mathrm{O}$ ligand ( $\mathrm{SCD} 4 \mathrm{OL})$ is associated with some pathobiological states. However, whether $\mathrm{SCD} 4 \mathrm{OL}$ in patients with chronic thromboembolic pulmonary hypertension (CTEPH) who underwent pulmonary endarterectomy (PEA) is associated with perioperative pulmonary hemodynamics and surgical outcomes has not been elucidated. Here we aimed to investigate whether SCD4OL is a useful serologic biomarker of poor surgical outcome of PEA in patients with $\mathrm{CTEPH}$.

Methods: Ninety patients with CTEPH who underwent PEA were enrolled. Independent preoperative parameters were examined, including $\mathrm{S} C D 40 \mathrm{~L}$ related to lower cardiac index (CI), higher pulmonary vascular resistance (PVR), and poor surgical outcomes after PEA, according to the multivariate logistic regression analysis. In addition, the area under the curve (AUC) value of $S C D 4 O L$ to predict poor surgical outcomes was compared with the AUCs of D-dimer and C-reactive protein (CRP). The generalizability of this study model was tested by a 5 -fold cross-validation analysis.

Results: Multivariate logistic regression analysis showed that high $\mathrm{SCD} 4 \mathrm{OL}$ level was related to postoperative lower $\mathrm{Cl}$, higher PVR, and poor surgical outcomes independent of other preoperative parameters. The AUC value of SCD4OL to predict poor surgical outcomes was higher than those of D-dimer and CRP. A SCD4OL cutoff value of $1.45 \mathrm{ng} / \mathrm{mL}$ predicted poor surgical outcomes with $79.3 \%$ sensitivity and $67.3 \%$ specificity. The 5 -fold cross-validation analysis showed the effectiveness of our model's performance.

Conclusions: Preoperative $\mathrm{SCD} 4 \mathrm{OL}$ level could be a promising serologic biomarker associated with poor surgical outcomes in CTEPH. In addition to known preoperative parameters, the biomarker might have the potential to identify patients at high risk of PEA, thereby reducing the mortality rates. (JTCVS Open 2021;8:618-29)

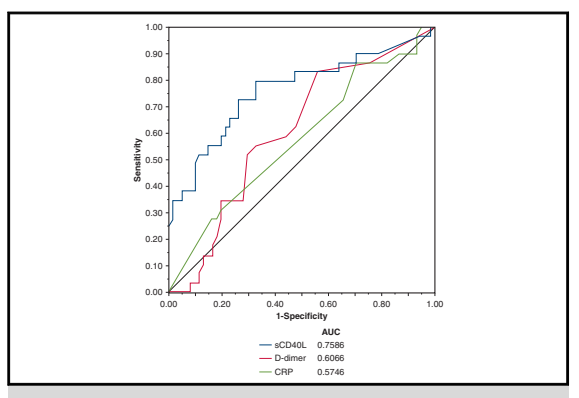

SCD4OL has a higher AUC for predicting poor surgical outcome in CTEPH compared with CRP or D-dimer.

CENTRAL MESSAGE

Preoperative soluble CD40 ligand

could be a promising serologic

biomarker associated with surgical

outcomes of pulmonary endarter-

ectomy in patients with chronic

thromboembolic pulmonary

hypertension.

\section{PERSPECTIVE}

High preoperative soluble CD40 ligand (sCD4OL) level was found to be related to poor surgical outcomes of pulmonary endarterectomy (PEA) independent of other preoperative parameters. Using $1.45 \mathrm{ng} / \mathrm{mL}$ as a cutoff value, the sensitivity and specificity for estimating poor surgical outcomes in patients with chronic thromboembolic pulmonary hypertension (CTEPH) were $79.3 \%$ and $67.3 \%$, respectively. In patients with $\mathrm{CTEPH}$, preoperative sCD4OL could be a promising serologic biomarker associated with surgical outcomes of PEA.

See Commentaries on pages 630 and 631 .

From the Departments of ${ }^{\mathrm{a}}$ Respirology, ${ }^{\mathrm{c}}$ Radiology, and ${ }^{\mathrm{d}}$ Cardiovascular Surgery, Graduate School of Medicine, Chiba University, Chiba, Japan; ' ${ }^{\mathrm{D}}$ Department of Respirology, Chibaken Saiseikai Narashino Hospital, Narashino, Japan; and ${ }^{\mathrm{e}}$ Department of Cardiovascular Surgery, Eastern Chiba Medical Center, Togane, Japan.

This study was supported by Japanese Ministry of Health, Labor and Welfare research grant specifically designated to the Respiratory Failure Research Group and Cardiovascular Diseases and the Pulmonary Hypertension Research Group from the Japan Agency for Medical Research and Development (16ek0109127h0002). The funding authority had no role in the study design, data collection or analysis, decision to publish, or manuscript preparation.
Received for publication Jan 27, 2021; revisions received Aug 12, 2021; accepted for publication Sept 9, 2021; available ahead of print Nov 6, 2021.

Address for reprints: Ayako Shigeta, MD, PhD, Department of Respirology, Graduate Japan (E-mail: aya0107@ chiba-u.jp).

2666-2736

Copyright (C 2021 The Author(s). Published by Elsevier Inc. on behalf of The American Association for Thoracic Surgery. This is an open access article under the CC BY-NC-ND license (http://creativecommons.org/licenses/by-nc-nd/4.0/).

https://doi.org/10.1016/j.xjon.2021.10.007 School of Medicine, Chiba University, 1-8-1 Inohana, Chuou-Ku Chiba 260-8670, 


$$
\begin{aligned}
& \text { Abbreviations and Acronyms } \\
& \begin{aligned}
\text { AUC } & =\text { area under the curve } \\
\text { BPA } & =\text { balloon pulmonary angioplasty } \\
\text { CD40L } & =\text { CD40 ligand } \\
\text { CI } & =\text { cardiac index } \\
\text { CRP } & =\text { C-reactive protein } \\
\text { CTEPH } & =\text { chronic thromboembolic pulmonary } \\
& \text { hypertension } \\
\text { PEA } & =\text { pulmonary endarterectomy } \\
\text { PH } & =\text { pulmonary hypertension } \\
\text { PVR } & =\text { pulmonary vascular resistance } \\
\text { RHC } & =\text { right heart catheterization } \\
\text { SCD40L } & =\text { soluble CD40 ligand }
\end{aligned}
\end{aligned}
$$

Video clip is available online.

Chronic thromboembolic pulmonary hypertension $(\mathrm{CTEPH})$ is a form of pulmonary hypertension $(\mathrm{PH})^{1}$ that develops as a complication of persistent chronic thrombi following acute pulmonary thromboembolism. Although the treatment options for CTEPH have expanded to include pulmonary endarterectomy (PEA), administration of soluble guanylate cyclase stimulators, and balloon pulmonary angioplasty (BPA), ${ }^{2,3}$ the only curative treatment for CTEPH is PEA, involving the surgical removal of organized thrombi with intima in the pulmonary artery. Effective PEA results in the normalization of hemodynamics with significant and immediate reductions in pulmonary vascular resistance (PVR) and pulmonary arterial pressure, as well as an increase in cardiac index (CI). The in-hospital mortality rate of PEA has been declining over time, owing to the increased experience of surgeons in performing such procedures and is currently reported to be $<5 \%$ in expert centers for PEA. ${ }^{4}$ However, even now, early postoperative morbidity and mortality may remain higher in PEA. Therefore, predicting the surgical outcome preoperatively is a challenging but essential step toward reducing morbidities. This is achieved by encouraging careful preparation or identifying alternative treatments for at-risk patients.

The CD40 ligand (CD40L) is a member of the tumor necrosis factor family. Recently, it has been recognized that the CD40L is related to the development of systemic vascular inflammation and induces the expression of inflammatory cytokines and molecules that help monocytes and leukocytes migrate into endothelial tissues. ${ }^{5,6}$ Studies on the cellular distribution of CD40L indicate that $>95 \%$ of circulating CD40L (soluble CD40L [sCD40L]) is derived from activated platelets. ${ }^{7}$ It is also known that high levels of sCD40L are associated with atherosclerosis and acute coronary syndrome ${ }^{8,9}$ and significantly correlated with the severity of clinical symptoms and hemodynamics in patients with chronic heart failure, suggesting that CD40L itself induces persistent tissue inflammation and remodeling of a failing myocardium. ${ }^{10}$

As noted above, several diseases related to inflammation and thrombosis are known to be associated with higher sCD40L levels. Previous studies have showed that vascular inflammation contributes to the pathogenesis of CTEPH. ${ }^{11,12}$ Moreover, excessive inflammation has been suggested to induce thrombus nonresolution, such as in the concept of "inflammatory thrombus." "11,13 However, whether CD40L gives rise to not only systemic, but also pulmonary, vascular inflammation has not been determined. In our previous study, we found that CD40L induced pulmonary microvascular endothelial cells to secrete inflammatory cytokines and growth factors in vitro. ${ }^{14}$ Furthermore, sCD40L levels are significantly higher in patients with idiopathic pulmonary arterial hypertension compared with controls. ${ }^{15}$ Moreover, patients with CTEPH are known to have highly activated platelets. ${ }^{16}$

In this study of patients with CTEPH who underwent PEA, we focused on SCD40L, which is known to be related to activated platelet activation, vascular inflammation, and thrombus nonresolution. This study aimed to identify any association between preoperative SCD40L level and the surgical outcomes of patients who underwent PEA for CTEPH.

\section{METHODS}

This study was approved by the Institutional Review Board of Chiba University Graduate School of Medicine (approval 826; October 12, 2020). According to Japanese legislation, informed consent is not required for the collection of retrospective data.

\section{Study Participants}

We enrolled 90 patients with CTEPH who underwent PEA at Chiba University Hospital, Chiba, Japan between August 2001 and October 2019. All patients were diagnosed with CTEPH by right heart catheterization (RHC), pulmonary angiography, lung perfusion scan, or computed tomography angiography. Patients who died in the hospital after PEA were excluded from the analysis of the postoperative parameters because they were not able to undergo postoperative RHC. The selection criteria for PEA were (1) both mean pulmonary arterial pressure $\geq 25 \mathrm{~mm} \mathrm{Hg}$ and calculated PVR $\geq 300$ dynes.s.cm ${ }^{-5}$ even after receipt of oral anticoagulant therapy for $>6$ months; (2) World Health Organization (WHO) functional class $\geq \mathrm{II}$; (3) presence of thrombus at the main, lobar, or segmental arteries confirmed by pulmonary angiography; and (4) absence of severe associated diseases. Eleven ageand sex-matched healthy subjects were enrolled as the control group.

\section{Operative Approach and Protocol}

We performed PEA with periods of hypothermic circulatory arrest according to the standard protocol developed by the University of California at San Diego group ${ }^{17}$ and used low-dose catecholamine postoperatively and diuretics aggressively to prevent reperfusion lung edema throughout the study period. ${ }^{18}$ 


\section{Subgroups}

To examine the relationship between the postoperative hemodynamics and the surgical outcome, we divided the 90 patients with CTEPH into 2 groups based on the postoperative CI. Patients with a CI $\geq 2.5 \mathrm{~L} / \mathrm{min} / \mathrm{m}^{2}$ were classified as the good postoperative CI group, and those with a $\mathrm{CI}<2.5 \mathrm{~L} / \mathrm{min} / \mathrm{m}^{2}$ were classified as the poor postoperative CI group. According to the 2015 European Society of Cardiology/European Respiratory Society PH guidelines, low CI $\left(<2.5 \mathrm{~L} / \mathrm{min} / \mathrm{m}^{2}\right)$ is associated with an intermediate or high mortality risk. ${ }^{1}$ Delcroix and colleagues ${ }^{19}$ suggested that the risk stratification for pulmonary arterial hypertension also may be applicable for patients with CTEPH, including post-PEA patients. ${ }^{19}$ Moreover, low postoperative CI is correlated with long-term survival of patients after PEA. ${ }^{20}$ Thus, we defined postoperative CI $<2.5 \mathrm{~L} / \mathrm{min} / \mathrm{m}^{2}$ as an index of poor postoperative cardiac outcome.

The patients were also divided into the good and poor postoperative PVR groups. Postoperative PVR $>500$ dynes.s. $\mathrm{cm}^{-5}$ has been identified as a strong predictor of mortality after PEA. ${ }^{17,21,22}$ The poor postoperative PVR group comprised patients who met at least 1 of the following conditions: (1) postoperative PVR $\geq 500$ dynes.s. $\mathrm{cm}^{-5}$ (based on published prognostic levels in CTEPH patients after PEA $^{23-25}$ ); (2) in-hospital mortality caused by persistent $\mathrm{PH}$; and (3) postoperative PVR level exceeding the preoperative PVR level. The remaining patients composed the good postoperative PVR group.

We defined poor surgical outcome in patients with CTEPH according to the following criteria: (1) poor postoperative CI; (2) poor postoperative PVR as mentioned above; and (3) all-cause in-hospital deaths.

\section{Measurement of Serum sCD40L, C-Reactive Protein, and D-Dimer}

All blood samples in the CTEPH group were collected during preoperative RHC and approved as part of a biorepository by the Institutional Review Board of Chiba University Graduate School of Medicine. All participants in the CTEPH group were treated with oral warfarin and were transitioned to intravenous heparin 2 days before RHC and blood sampling. Serum sCD40L was measured using a commercial sCD40L enzyme-linked immunosorbent assay kit (R\&D Systems, Minneapolis, Minn) by SRL (Tokyo, Japan). Serum C-reactive protein (CRP) and D-dimer were measured by immune nephelometry.

\section{RHC}

Patients underwent RHC for both the preoperative evaluation (preoperative RHC) and the evaluation of PEA outcome (postoperative RHC) when they were in stable conditions. The mean time from preoperative RHC to PEA was $120.0 \pm 182.7$ days, and the mean time from PEA to postoperative RHC was $42.6 \pm 14.0$ days.

\section{Logistic Regression Analysis}

Univariate logistic regression analysis was performed to examine the correlation between preoperative parameters and surgical outcomes. Then multivariate logistic regression analysis was performed to identify independent preoperative parameters related to surgical outcomes, such as low postoperative CI, persistent $\mathrm{PH}$, and in-hospital mortality.

\section{Receiver Operating Characteristic Curves}

Receiver operating characteristic curves were constructed to compare the area under the curve (AUC) of SCD40L with the AUCs of CRP and D-dimer. The optimal sCD40L threshold value was then determined, to estimate its sensitivity and specificity for predicting poor surgical outcomes. In addition, 5-fold cross-validation was performed to evaluate the predictive performance of sCD40L. Moreover, a random forest plot was used to evaluate the importance of the variables in predicting prognosis. The decrease in Gini impurity was calculated 5 times in
TABLE 1. Characteristics of the patients with CTEPH $(\mathbf{N}=90)$

\begin{tabular}{lcc}
\hline \multicolumn{1}{c}{ Characteristic } & Value \\
\hline Sex, male/female, $\mathrm{n}$ & $28 / 62$ \\
Age, $\mathrm{y}$, mean $\pm \mathrm{SD}$ & $59.2 \pm 1.1$ \\
WHO functional class, I/II/III/IV, $\mathrm{n}$ & \multicolumn{2}{c}{$0 / 44 / 42 / 4$} \\
Jamieson classification, I/II/III/IV, $\mathrm{n}$ & \multicolumn{2}{c}{$49 / 25 / 16 / 0$} \\
Operative mortality, $\mathrm{n}$ & \multicolumn{2}{c}{6} \\
Perioperative parameters, mean $\pm \mathrm{SD}$ & Preoperative & Postoperative \\
RAP, mm Hg & $6.1 \pm 3.5$ & $4.7 \pm 2.9$ \\
mPAP, mm Hg & $46.1 \pm 9.5$ & $24.9 \pm 9.0$ \\
PVR, dynes.s.cm ${ }^{-5}$ & $773 \pm 289$ & $306 \pm 186$ \\
CI, $\mathrm{L} / \mathrm{min} / \mathrm{m}^{2}$ & $2.6 \pm 0.6$ & $3.0 \pm 0.5$ \\
\hline
\end{tabular}

$S D$, Standard deviation; $W H O$, World Health Organization; $R A P$, right atrial pressure; $m P A P$, mean pulmonary arterial pressure; $P V R$, pulmonary vascular resistance; $C I$, cardiac index.

5-fold cross-validation, and the variables were ranked. ${ }^{26}$ The AUC of the random forest after 5-fold cross-validation was also calculated.

\section{Statistical Analysis}

Our results are expressed as mean $\pm \mathrm{SD}$. Data analysis was done with JMP 15.0 software (Japanese version, SAS Institute, Tokyo, Japan) and $\mathrm{R}$ version 3.51 (R Foundation for Statistical Computing, Vienna, Austria). A $P$ value $<.05$ was considered statistically significant. Group comparisons were done using the $\chi^{2}$ test. Comparisons of the differences between two groups were done using the Mann-Whitney $U$ test. Correlations between serum $\mathrm{SCD} 40 \mathrm{~L}$ level and perioperative parameters were assessed by Spearman's correlation coefficient.

\section{RESULTS}

\section{sCD40L in CTEPH and Control Patients}

The CTEPH group comprised 90 patients who underwent PEA during the study period. The clinical characteristics

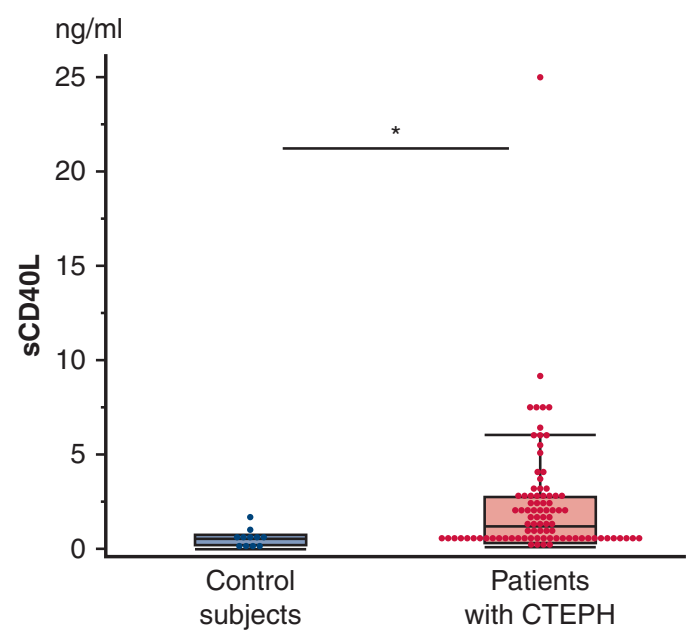

FIGURE 1. The difference in soluble CD40 ligand ( $s C D 4 O L)$ between control subjects and patients with chronic thromboembolic pulmonary hypertension $(C T E P H)$. sCD40L is significantly higher in patients with CTEPH compared with controls (mean, $2.2 \pm 3.1 \mathrm{ng} / \mathrm{mL}$ vs $0.5 \pm 0.5 \mathrm{ng} / \mathrm{mL} ; P=.009)$. 


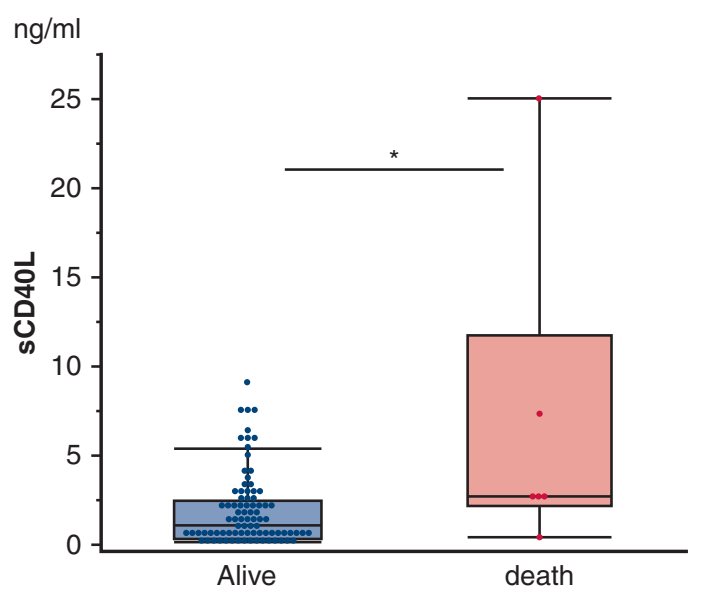

A

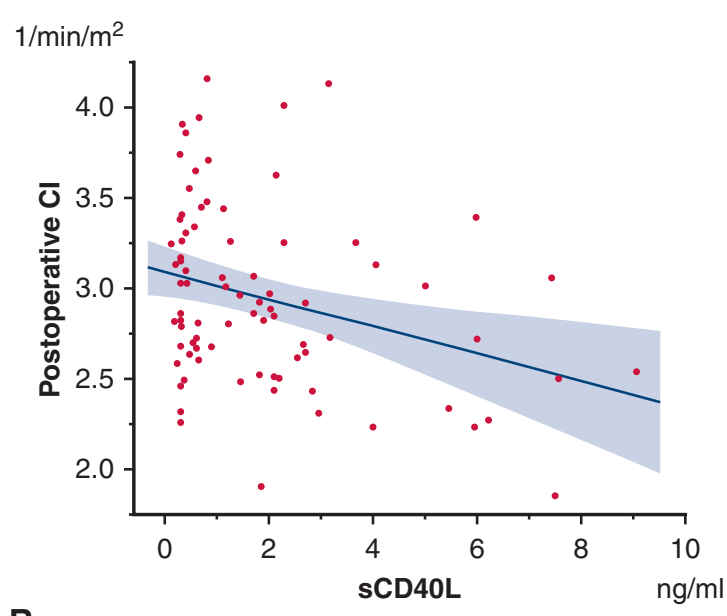

B

FIGURE 2. A, Preoperative soluble CD40 ligand ( $s C D 4 O L)$ in patients with chronic thromboembolic pulmonary hypertension (CTEPH) who are alive compared with those who died in the hospital. "alive" on the horizontal line indicates the subjects without operation-related death, and "death" indicates the subjects with operation-related death. The upper and lower borders of the box represent the upper and lower quartiles. The middle horizontal line represents the median. The upper and lower whiskers represent the maximum and minimum values of nonoutliers. Extra dots represent outliers. Preoperative sCD40L is significantly higher in the patients with operation-related deaths (mean, $6.8 \pm 9.2 \mathrm{ng} / \mathrm{mL}$ vs $1.9 \pm 2.1 \mathrm{ng} / \mathrm{mL} ; P=.02$ ). B, Correlation between preoperative sCD40L and postoperative cardiac index $(C I)$. Preoperative sCD40L is correlated with postoperative $\mathrm{CI}(P=.02)$.

and perioperative parameters of the CTEPH group are shown in Table 1. As shown in Figure 1, serum sCD40L levels were significantly higher in the CTEPH group compared with control group (mean, $2.2 \pm 3.1 \mathrm{ng} / \mathrm{mL}$ vs $0.5 \pm 0.5 \mathrm{ng} / \mathrm{mL} ; P=.009)$.

\section{sCD40L and Perioperative Parameters}

SCD40L levels in all groups subdivided by perioperative parameters are shown in Table E1. As shown in Figure 2, A, sCD40L levels were significantly higher in the patients with CTEPH who died in the hospital compared with those who did not $(P=.02)$. Table E2 shows correlations between sCD40L levels and perioperative parameters. Six patients who died in the hospital after PEA were excluded from the postoperative analysis. SCD40L level had a negative correlation with postoperative CI $(P=.02)$, but the other perioperative parameters did not (Figure 2, B).

\section{sCD40L and Surgical Outcome}

As shown in Table E3, univariate logistic regression analysis revealed that among the preoperative parameters, only SCD40L level was significantly correlated with poor postoperative CI and poor surgical outcomes, and that SCD40L and BNP were significantly correlated with poor postoperative PVR. Furthermore, as shown in Table 2, multivariate logistic analysis performed using sCD40L and BNP (identified as significant variables in the univariate logistic analysis) and PVR (previously reported to be related to surgical outcomes) revealed that only sCD40L level was significantly correlated with poor postoperative CI, poor postoperative PVR, and poor surgical outcomes. This suggested that sCD40L could be an independent preoperative parameter related to poor surgical outcomes, including low cardiac function, persistent $\mathrm{PH}$, and in-hospital mortality.

\section{Comparisons of SCD40L and Other Markers}

Finally, to evaluate whether preoperative SCD40L would be a useful serological biomarker for estimating the surgical outcome, we constructed a receiver operating characteristic curve. Moreover, we compared the AUC of preoperative sCD40L with those of preoperative CRP and D-dimer level

TABLE 2. Multivariate logistic analysis of preoperative parameters for the risk of poor postoperative outcome subgroups

\begin{tabular}{|c|c|c|c|c|c|c|}
\hline \multirow[b]{2}{*}{ Preoperative parameter } & \multicolumn{2}{|c|}{$\begin{array}{c}\text { Post-CI subgroup } \\
(\operatorname{good} / \text { poor, } n=74 / 16)\end{array}$} & \multicolumn{2}{|c|}{$\begin{array}{l}\text { Post-PVR subgroup } \\
(\operatorname{good} / \text { poor, } \mathbf{n}=77 / 13)\end{array}$} & \multicolumn{2}{|c|}{$\begin{array}{c}\text { Surgical outcome } \\
(\mathrm{good} / \text { poor, } n=61 / 29)\end{array}$} \\
\hline & OR $(95 \%$ CI $)$ & $P$ & OR $(95 \%$ CI $)$ & $P$ & $\overline{\text { OR }(95 \% \text { CI })}$ & $P$ \\
\hline $\mathrm{sCD} 40 \mathrm{~L}, \mathrm{ng} / \mathrm{mL}$ & $1.49(1.2-1.9)$ & .001 & $1.55(1.2-2.0)$ & .001 & $1.77(1.3-2.4)$ & $<.0001$ \\
\hline PVR, dynes.s.cm ${ }^{-5}$ & $0.99(0.9-1.0)$ & .06 & $1.00(0.9-1.0)$ & .92 & $0.99(0.9-1.0)$ & .33 \\
\hline BNP, $p g / m L$ & $1.00(0.9-1.0)$ & .15 & $1.00(0.9-1.0)$ & .07 & $1.00(0.9-1.0)$ & .14 \\
\hline
\end{tabular}

$C I$, Cardiac index; $P V R$, pulmonary vascular resistance; $O R$, odds ratio; $C I$, confidence interval; $S C D 4 O L$, soluble CD40 ligand; $B N P$, brain natriuretic peptide. 


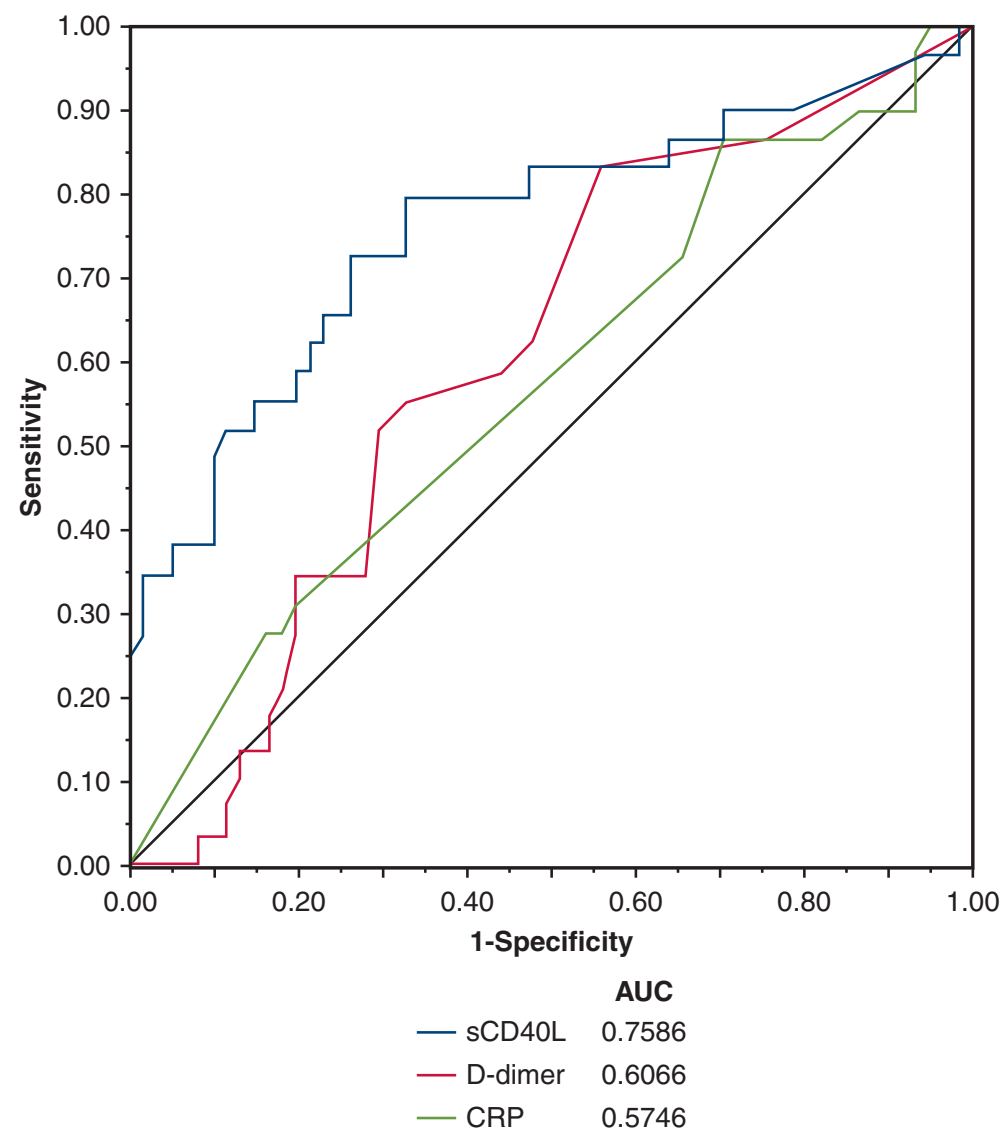

FIGURE 3. Area under the curve $(A U C)$ for predicting poor surgical outcome by preoperative soluble CD40 ligand $(s C D 4 O L)$, C-reactive protein $(C R P)$, and D-dimer levels. Preoperative SCD40L has a higher AUC value for predicting poor surgical outcome in chronic thromboembolic pulmonary hypertension than preoperative CRP or D-dimer.

reported as biomarkers related to pathogenesis and clinical outcome of CTEPH. ${ }^{27-29}$ As shown in Figure 3, sCD40L had a higher AUC for predicting poor surgical outcome compared with CRP and D-dimer (AUC of 0.76, 0.57, and 0.60 , respectively). The $95 \%$ CI of AUC straddled 0.50 in both CRP and D-dimer, meaning they were not significant predictors for surgical outcome.

The cutoff value for $\mathrm{sCD} 40 \mathrm{~L}$ was $1.45 \mathrm{ng} / \mathrm{mL}$, which had maximum sensitivity and specificity for dividing patients into the good and poor surgical outcome groups. As shown in Table 3, using this cutoff value, the sensitivity and specificity for predicting poor surgical outcomes were $79.3 \%$ and $67.3 \%$, respectively. An additional 5-fold cross-validation analysis proved the excellent predictive performance of sCD40L in this study. Our findings indicate that using preoperative $\mathrm{SCD} 40 \mathrm{~L}$ better distinguishes patients who had a poor surgical outcome after PEA from those who did not have a poor outcome compared with using CRP and D-dimer as predictive biomarkers of CTEPH. Moreover, sCD40L was the most important variable in all 5 iterations of the 5-fold cross-validation of our random forest plot (Table E4). The AUC of the random forest after the 5-fold cross-validation was 0.71 (Table 3).

TABLE 3. Usefulness of SCD40L in predicting poor surgical outcomes

\begin{tabular}{lcccccc}
\hline Parameter & Cutoff value & AUC $(\mathbf{9 5} \% \mathbf{C I})$ & Sensitivity, $\% \mathbf{( 9 5} \% \mathbf{C I})$ & Specificity, $\%, \mathbf{9 5} \% \mathbf{C I})$ & OR $(\mathbf{9 5} \% \mathbf{C I})$ & $\boldsymbol{P}$ value \\
\hline sCD40L & 1.45 & $0.76(0.64-0.88)$ & $79.3(55.2-93.1)$ & $67.3(19.7-83.6)$ & $7.0(2.6-20.2)$ & $<.0001$ \\
& & $0.73(0.60-0.86)^{*}$ & $79.3(55.2-89.7)$ & $67.2(20.2-83.6)$ & & \\
& & $0.71(0.59-0.83) \dagger$ & $51.7(27.6-69.0)$ & $85.3(50.8-95.1)$ & & \\
D-dimer & 0.30 & $0.61(0.49-0.73)$ & $82.7(52.6-94.0)$ & $44.3(12.3-57.8)$ & $3.0(1.1-9.2)$ & .03 \\
CRP & 0.20 & $0.57(0.45-0.70)$ & $29.6(3.3-42.0)$ & $86.2(58.6-96.6)$ & $0.8(0.3-2.1)$ & .62 \\
\hline
\end{tabular}

$A U C$, Area under the curve; $C I$, confidence interval; $O R$, odds ratio; $S C D 40 L$, soluble CD40 ligand; $C R P$, C-reactive protein. *Logistic regression after 5-fold cross-validation. $\dagger$ Random forest after 5 -fold cross-validation. 
Following the published paper, ${ }^{29}$ we also examined the association of preoperative $\mathrm{SCD} 40 \mathrm{~L}$ with cardiopulmonary bypass time, circulation arrest time, surgery time, aortic cross clamping time, catecholamine and postoperative extracorporeal membrane oxygenation support. Using the median preoperative sCD40L level, we divided the patients into 2 groups, $<121 \mathrm{ng} / \mathrm{mL}$ and $>1.21 \mathrm{ng} / \mathrm{mL}$, and compared these parameters between the 2 groups. As shown in Table E5, there were no significant differences in intraoperative variables or in the ratio of subjects with postoperative extracorporeal membrane oxygenation support between the 2 groups. With regard to catecholamine requirements, patients with $>1.21 \mathrm{ng} / \mathrm{mL}$ required significantly longer catecholamine support after PEA than those with $<1.21 \mathrm{ng} / \mathrm{mL}$ (mean, $2.6 \pm 2.0$ days vs $1.5 \pm 0.9$ days; $P=.007)$.

\section{DISCUSSION}

In the present study, we found SCD40L level to be a useful serologic biomarker in CTEPH associated with morbidities resulting from low postoperative $\mathrm{CI}$, persistent $\mathrm{PH}$, and in-hospital mortality (Video 1). It has been reported that the surgical outcome of PEA depends on preoperative PVR, New York Heart Association functional class, and level of experience of the surgical team. ${ }^{4,30}$ Based on our present results, preoperative sCD40L level in patients with CTEPH could be a promising predictor for surgical outcomes in addition to its well-known utility for preoperative parameters. Methods, representative results, and interpretation of these results are summarized in Figure 4.

Ueland and colleagues ${ }^{10}$ suggested a pathogenic role for enhanced CD40-CD40L interactions in human myocardial failure. In the present study, there was a negative correlation between $\mathrm{SCD} 40 \mathrm{~L}$ level and postoperative $\mathrm{CI}$, although no

\section{Characteristics of patients with CTEPH (n=90)}

\begin{tabular}{|c|c|c|}
\hline (male : female) & \multicolumn{2}{|c|}{$28: 62$} \\
\hline (years) & \multicolumn{2}{|c|}{$59.2 \pm 1.1$} \\
\hline WHO functional class $\quad$ (I : II : III : IV) & \multicolumn{2}{|c|}{$0: 44: 42: 4$} \\
\hline Jamieson classification (I : II : III : IV) & \multicolumn{2}{|c|}{$49: 25: 16: 0$} \\
\hline \multirow[t]{2}{*}{ operative mortality } & \multicolumn{2}{|c|}{6} \\
\hline & preoperative & postoperative \\
\hline RAP (mmHg) & $6.1 \pm 3.5$ & $4.7 \pm 2.9$ \\
\hline mPAP (mmHg) & $46.1 \pm 9.5$ & $24.9 \pm 9.0$ \\
\hline PVR (dynes.sec.cm ${ }^{-5}$ ) & $773 \pm 289$ & $306 \pm 186$ \\
\hline $\mathrm{Cl}\left(\mathrm{L} / \mathrm{min} / \mathrm{m}^{2}\right)$ & $2.6 \pm 0.6$ & $3.0 \pm 0.5$ \\
\hline
\end{tabular}

VIDEO 1. Dr Ayako Shigeta discusses preoperative soluble CD40 ligand in patients with chronic thromboembolic pulmonary hypertension as a promising biomarker associated with poor surgical outcome of pulmonary endarterectomy. Video available at: https://www.jtcvs.org/article/S26662736(21)00357-0/fulltext. association with preoperative CI was observed, suggesting that a higher sCD40L level might indicate an occult cardiac dysfunction, which is difficult to estimate using the preoperative CI value.

Jamieson and associates reported that postoperative PVR $>500$ dynes.sec. $\mathrm{cm}^{-5}$ was strongly associated with an increased risk of mortality in patients with CTEPH, and the strongest predictor of mortality after PEA is postoperative residual PVR, which indicates inaccessible distal obstruction. ${ }^{17}$ Madani and colleagues also reported that the postoperative mortality rates were $10.3 \%$ in CTEPH patients with a postoperative PVR $>500$ dynes.s.cm ${ }^{-5}$ and only $0.9 \%$ in CTEPH patients with a postoperative PVR $<500$ dynes.s.cm ${ }^{-5} \cdot{ }^{21,22}$ Therefore, as shown in this study, preoperative sCD40L level associated with persistent PH could help to estimate the surgical mortality of patients with CTEPH.

To examine the association between SCD40L level and the morphology of thromboembolic disease, we investigated the association between preoperative $\mathrm{sCD} 40 \mathrm{~L}$ and the Jamieson classification. No patients with type $4 \mathrm{CTEPH}$ were identified in this study. As shown in Figure E1, there was a tendency toward differences in mean SCD40L level among the Jamieson classification types (type 1, $2.6 \pm 3.9 \mathrm{ng} / \mathrm{mL}$; type $2,1.0 \pm 2.0 \mathrm{ng} / \mathrm{mL}$; type 3 , $3.1 \pm 2.5 \mathrm{ng} / \mathrm{mL} ; P=.06)$. Post hoc tests showed relatively higher sCD40L levels in type 1 and type 3 than in type 2 . sCD40L could be up-regulated on the platelets in a fresh thrombus ${ }^{31}$ which might be the reason for higher sCD40L in patients with type 1 CTEPH (fresh thrombus in the main lobar pulmonary arteries). In addition, inflammation from the fresh thrombus might weaken the organized thrombus, resulting in inadequate removal and residual PH. Type 3 CTEPH (ie, disease within the distal segmental arteries only) has been known to correlate with vascular inflammation and in situ thrombus, ${ }^{32}$ which might be the reason for higher sCD40L levels in patients with type 3 CTEPH. Vascular inflammation and in situ thrombus on the distal side from the surgically reachable site might be associated with residual $\mathrm{PH}$. These results suggest the involvement of sCD40L in the pathogenesis of CTEPH, such as small vascular inflammation and thrombosis nonresolution.

Preoperative sCD40L was found to be a better predictive serologic biomarker than CRP or D-dimer in CTEPH patients who underwent PEA. CRP is widely recognized as a biomarker of inflammatory diseases, including CTEPH. It has been reported that CRP is elevated in blood and thrombus tissue of patients with $\mathrm{CTEPH}^{33}$ and contributes to the pathogenesis of CTEPH through the promotion of pulmonary vascular remodeling, endothelial dysfunction, and in situ thrombosis. ${ }^{12}$ D-dimer, a degradation product of cross-linked fibrin, is widely used to help rule out the presence of thrombi. Elevated D-dimer levels in the blood 
Prognostic value of preoperative SCD40L for surgical outcome in CTEPH patients with PEA
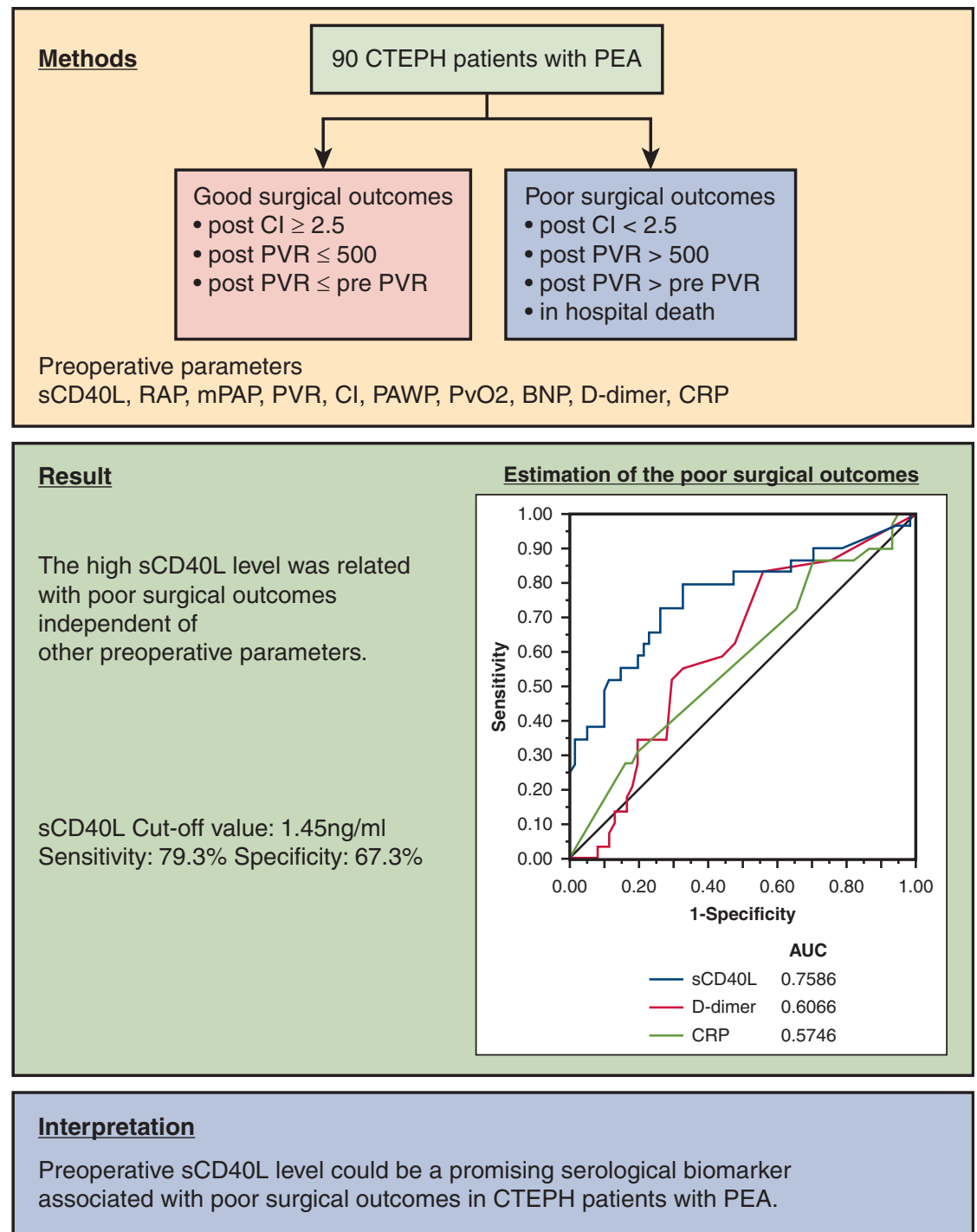

FIGURE 4. Ninety patients with chronic thromboembolic pulmonary hypertension $(C T E P H)$ who underwent pulmonary endarterectomy $(P E A)$ were divided into good and poor surgical outcome groups. We examined the relationship between preoperative soluble CD40 ligand $(s C D 40 L)$ level and surgical outcome. A high preoperative sCD40L level was correlated with poor surgical outcome independent of other preoperative parameters. The area under the blood concentration time curve AUC value of preoperative SCD40L for predicting poor surgical outcome was higher than that of D-dimer and CRP. Preoperative sCD40L level of CTEPH patients could be a promising biomarker related to surgical outcome of PEA. CI, Cardiac index; PVR, pulmonary vascular resistance; $R A P$, right atrial pressure; $m P A P$, mean pulmonary arterial pressure; $P A W P$, pulmonary artery wedge pressure; $P v O_{2}$, mixed venous oxygen pressure; $B N P$, brain natriuretic peptide; $C R P$, C-reactive protein; $A U C$, area under the curve.

of patients with CTEPH have been reported and correlated with hemodynamics and World Health Organization functional status of CTEPH. ${ }^{28}$ Skoro-Sajer and colleagues ${ }^{28}$ showed that CRP and D-dimer were independent and significant predictors of outcome in CTEPH. Arthur Ataam and colleagues ${ }^{29}$ showed that an increase of $\geq 10 \mathrm{mg} / \mathrm{L}$ over preoperative CRP level was independently related to adverse early outcomes post-PEA. In the present study, we found that preoperative $\mathrm{sCD} 40 \mathrm{~L}$ could be more useful than CRP and D-dimer for distinguishing patients who had poor surgical outcomes from those who did not have poor outcomes. In addition, patients with high preoperative sCD40L levels required prolonged catecholamine support immediately after PEA. This may indicate that the strength of $\mathrm{SCD} 40 \mathrm{~L}$ is related to both inflammation and thrombus formation involved in the pathogenesis of CTEPH and the hemodynamic instability just after PEA, not to just one or the other. 
In this study, the statistical outlier for sCD40L level was $>6.01 \mathrm{ng} / \mathrm{mL}$. Seven patients had a statistical outlier value of sCD40L, recognized as extremely high SCD40L levels. Six of these 7 patients had a poor surgical outcome, including post-PVR $>500$ dynes.s. $\mathrm{cm}^{-5}(\mathrm{n}=3)$, pre-PVR value below postoperative PVR value $(n=1)$, surgical death with persistent $\mathrm{PH}(\mathrm{n}=1)$, and surgical death with recurrent thrombosis $(\mathrm{n}=1)$. Higher sCD40L levels might be related to severe small vessel disease and excessive platelet activation, resulting in persistent $\mathrm{PH}$ and recurrent thrombosis. Based on these results, we propose that close monitoring for recurrent thrombosis and postoperative complications is essential for patients with extremely high sCD40L levels.

Our study has several limitations. First, this was a singlecenter study with a small patient cohort over a 19-year period, and there could be issues of bias in terms of patient selection and changes in practice during the study period. In addition, the number of patients in each subgroup was too small to consider the differences in SCD40L in the low CI group, high PVR group, and poor surgical outcome group. Moreover, further study is needed to validate the predictability of sCD40L for surgical outcome using data from other centers. Therefore, from this study, it can be said that preoperative SCD40L of patients with CTEPH is related to surgical outcome, but cannot be said to be a predictor of poor surgical outcome. Second, preoperative treatment with pulmonary vasodilators might have influenced sCD40L levels. Third, sensitivity and specificity based on the cutoff value of $\mathrm{SCD} 40 \mathrm{~L}$ might change depending on the surgical experience and the time from blood collection date to PEA surgery. Fourth, in this study, we demonstrated the utility of measuring preoperative sCD40L in patients with CTEPH associated with surgical outcomes, but we could not explain why a higher sCD40L level was associated with lower postoperative CI, poor postoperative PVR, and in-hospital mortality. Future studies are needed to understand the implications and impact of higher sCD40L levels in patients with CTEPH for establishing the credibility of sCD40L as a potential biomarker.

\section{CONCLUSIONS}

Preoperative sCD40L level may be a promising biomarker for identifying CTEPH patients at increased risk for residual $\mathrm{PH}$ and mortality after PEA, independent of preoperative hemodynamic severity. Therefore, a high CD40 level in CTEPH patients may be useful for identifying patients at risk of postoperative high PVR and low CI, independent of preoperative hemodynamic severity, suggesting the need for more careful management of patients with high preoperative CD40L to avoid hemodynamic compromise after PEA. Furthermore, operability must be assessed more carefully when severely compromised patients with heart failure and high PVR also have a high level of sCD40L.

\section{Conflict of Interest Statement}

The authors reported no conflicts of interest.

The Journal policy requires editors and reviewers to disclose conflicts of interest and to decline handling or reviewing manuscripts for which they may have a conflict of interest. The editors and reviewers of this article have no conflicts of interest.

We thank Editage for the English language editing.

\section{References}

1. Galiè N, Humbert M, Vachiery JL, Gibbs S, Lang I, Torbicki A, et al. 2015 ESC/ ERS guidelines for the diagnosis and treatment of pulmonary hypertension: the Joint Task Force for the Diagnosis and Treatment of Pulmonary Hypertension of the European Society of Cardiology (ESC) and the European Respiratory Society (ERS): Endorsed by: Association for European Paediatric and Congenital Cardiology (AEPC), International Society for Heart and Lung Transplantation (ISHLT). Eur Respir J. 2015;46:903-75.

2. Bouvaist H, Thony F, Jondot M, Camara B, Jais X, Pison C. Balloon pulmonary angioplasty in a patient with chronic thromboembolic pulmonary hypertension. Eur Respir Rev. 2014;23:393-5.

3. Kim NH, Delcroix M, Jenkins DP, Channick R, Dartevelle P, Jansa P, et al. Chronic thromboembolic pulmonary hypertension. J Am Coll Cardiol. 2013; 62(25 Suppl):D92-9

4. Jenkins D, Madani M, Fadel E, D’Armini AM, Mayer E. Pulmonary endarterectomy in the management of chronic thromboembolic pulmonary hypertension. Eur Respir Rev. 2017;26:160111.

5. Hassan GS, Merhi Y, Mourad WM. CD154 and its receptors in inflammatory vascular pathologies. Trends Immunol. 2009;30:165-72.

6. Thienel U, Loike J, Yellin MJ. CD154 (CD40L) induces human endothelial cell chemokine production and migration of leukocyte subsets. Cell Immunol. 1999; 198:87-95.

7. Anand SX, Viles-Gonzalez JF, Badimon JJ, Cavusoglu E, Marmur JD. Membrane-associated CD40L and SCD40L in atherothrombotic disease. Thromb Haemost. 2003;90:377-84.

8. Aukrust P, Müller F, Ueland T, Berget T, Aaser E, Brunsvig A, et al. Enhanced levels of soluble and membrane-bound CD40 ligand in patients with unstable angina. Possible reflection of $\mathrm{T}$ lymphocyte and platelet involvement in the pathogenesis of acute coronary syndromes. Circulation. 1999;100:614-20.

9. Conde ID, Kleiman NS. Soluble CD40 ligand in acute coronary syndromes. $N$ Engl J Med. 2003;348:2575-7; author reply 2575-7.

10. Ueland T, Aukrust P, Yndestad A, Otterdal K, Frøland SS, Dickstein K, et al Soluble CD40 ligand in acute and chronic heart failure. Eur Heart J. 2005;26: 1101-7.

11. Lang IM, Pesavento R, Bonderman D, Yuan JX. Risk factors and basic mechanisms of chronic thromboembolic pulmonary hypertension: a current understanding. Eur Respir J. 2013;41:462-8.

12. Wynants M, Quarck R, Ronisz A, Alfaro-Moreno E, Van Raemdonck D Meyns B, et al. Effects of C-reactive protein on human pulmonary vascular cells in chronic thromboembolic pulmonary hypertension. Eur Respir J. 2012;40: 886-94.

13. Matthews DT, Hemnes AR. Current concepts in the pathogenesis of chronic thromboembolic pulmonary hypertension. Pulm Circ. 2016;6:145-54.

14. Shigeta A, Tada Y, Wang JY, Ishizaki S, Tsuyusaki J, Yamauchi K, et al. CD40 amplifies Fas-mediated apoptosis: a mechanism contributing to emphysema. Am J Physiol Lung Cell Mol Physiol. 2012;303:L141-51.

15. Damås JK, Otterdal K, Yndestad A, Aass H, Solum NO, Frøland SS, et al. Soluble CD40 ligand in pulmonary arterial hypertension: possible pathogenic role of the interaction between platelets and endothelial cells. Circulation. 2004;110: 999-1005.

16. Yaoita N, Shirakawa R, Fukumoto Y, Sugimura K, Miyata S, Miura Y, et al. Platelets are highly activated in patients of chronic thromboembolic pulmonary hypertension. Arterioscler Thromb Vasc Biol. 2014;34:2486-94. 
17. Jamieson SW, Kapelanski DP, Sakakibara N, Manecke GR, Thistlethwaite PA, Kerr KM, et al. Pulmonary endarterectomy: experience and lessons learned in 1,500 cases. Ann Thorac Surg. 2003;76:1457-62; discussion 1462-4.

18. Mares P, Gilbert TB, Tschernko EM, Hiesmayr M, Muhm M, Herneth A, et al. Pulmonary artery thromboendarterectomy: a comparison of two different postoperative treatment strategies. Anesth Analg. 2000;90:267-73.

19. Delcroix M, Staehler G, Gall H, Grünig E, Held M, Halank M, et al. Risk assessment in medically treated chronic thromboembolic pulmonary hypertension patients. Eur Respir J. 2018;52:1800248.

20. Cannon JE, Su L, Kiely DG, Page K, Toshner M, Swietlik E, et al. Dynamic risk stratification of patient long-term outcome after pulmonary endarterectomy: results from the United Kingdom National Cohort. Circulation. 2016;133: 1761-71.

21. Madani MM, Auger WR, Pretorius V, Sakakibara N, Kerr KM, Kim NH, et al. Pulmonary endarterectomy: recent changes in a single institution's experience of more than 2,700 patients. Ann Thorac Surg. 2012;94:97-103; discussion 103.

22. Mayer E, Jenkins D, Lindner J, D’Armini A, Kloek J, Meyns B, et al. Surgical management and outcome of patients with chronic thromboembolic pulmonary hypertension: results from an International Prospective Registry. J Thorac Cardiovasc Surg. 2011;141:702-10.

23. Nickel N, Golpon H, Greer M, Knudsen L, Olsson K, Westerkamp V, et al. The prognostic impact of follow-up assessments in patients with idiopathic pulmonary arterial hypertension. Eur Respir J. 2012;39:589-96.

24. Riedel M, Stanek V, Widimsky J, Prerovsky I. Long-term follow-up of patients with pulmonary thromboembolism. Late prognosis and evolution of hemodynamic and respiratory data. Chest. 1982;81:151-8.

25. D’Armini AM, Ghofrani HA, Kim NH, Mayer E, Morsolini M, PulidoZamudio $\mathrm{T}$, et al. Use of responder threshold criteria to evaluate the response to treatment in the phase III CHEST-1 study. J Heart Lung Transplant. 2015; $34: 348-55$.
26. Witten IH, Frank E, Hall MA, Pal C. Data Mining: Practical Machine Learning Tools and Techniques. $4^{\text {th }}$ ed. Elsevier: Morgan Kaufmann; 2016.

27. Zhang M, Zhang Y, Pang W, Zhai Z, Wang C. Circulating biomarkers in chronic thromboembolic pulmonary hypertension. Pulm Circ. 2019;9: 2045894019844480.

28. Skoro-Sajer N, Gerges C, Gerges M, Klepetko W, Celermajer DS, Lang IM, et al. Usefulness of thrombosis and inflammation biomarkers in chronic thromboembolic pulmonary hypertension-sampling plasma and surgical specimens. J Heart Lung Transplant. 2018;37:1067-74.

29. Arthur Ataam J, Amsallem M, Guihaire J, Haddad F, Lamrani L, Stephan F, et al. Preoperative C-reactive protein predicts early postoperative outcomes after pulmonary endarterectomy in patients with chronic thromboembolic pulmonary hypertension. J Thorac Cardiovasc Surg. 2021;161:1532-42.e5.

30. Delcroix M, Lang I, Pepke-Zaba J, Jansa P, D’Armini AM, Snijder R, et al. Longterm outcome of patients with chronic thromboembolic pulmonary hypertension: results from an International Prospective Registry. Circulation. 2016;133: 859-71.

31. Henn V, Slupsky JR, Gräfe M, Anagnostopoulos I, Förster R, Müller-Berghaus G, et al. CD40 ligand on activated platelets triggers an inflammatory reaction of endothelial cells. Nature. 1998;391:591-4.

32. Quarck R, Wynants M, Verbeken E, Meyns B, Delcroix M. Contribution of inflammation and impaired angiogenesis to the pathobiology of chronic thromboembolic pulmonary hypertension. Eur Respir J. 2015;46:431-43.

33. Quarck R, Nawrot T, Meyns B, Delcroix M. C-reactive protein: a new predictor of adverse outcome in pulmonary arterial hypertension. J Am Coll Cardiol. 2009; 53:1211-8.

Key Words: soluble CD40 ligand, chronic thromboembolic pulmonary hypertension, pulmonary endarterectomy 


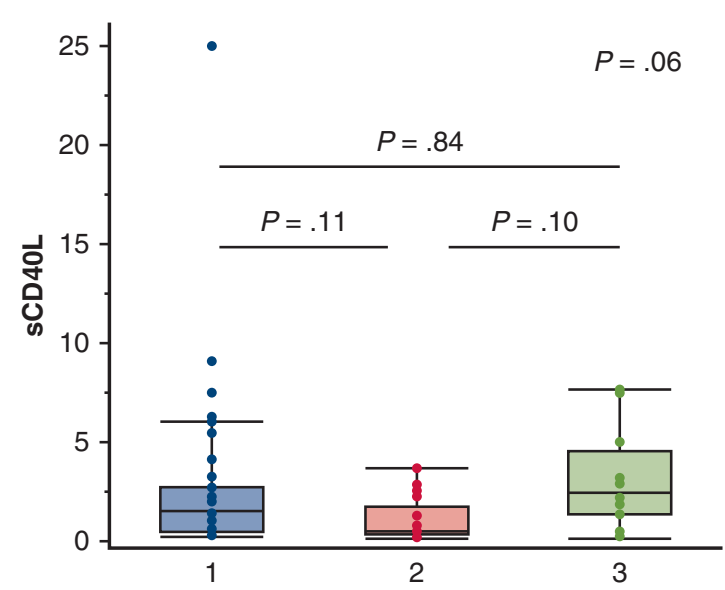

FIGURE E1. The comparison of preoperative SCD40L among Jamieson classification. Patients with Jamieson classification type 1 and type 3 had higher SCD40L than that in patients with Jamieson classification type 2. $s C D 40 L$, Soluble CD40 ligand.

TABLE E1. sCD40L value in each group subdivided by perioperative parameters

\begin{tabular}{lcc}
\hline \multicolumn{1}{c}{ Parameter } & sCD40L, ng/mL, mean \pm SD & $P$ value \\
\hline Sex, male/female & $3.0 \pm 4.7 / 1.8 \pm 2.1$ & .15 \\
WHO functional class II/III/IV & $1.8 \pm 1.9 / 1.8 \pm 2.0 / 9.7 \pm 10.6$ & .12 \\
Jamieson classification type 1/2/3 & $2.6 \pm 3.9 / 1.0 \pm 2.0 / 3.1 \pm 2.5$ & .06 \\
In-hospital mortality, alive/dead & $1.8 \pm 2.0 / 6.8 \pm 9.1$ & .02 \\
\hline
\end{tabular}

$s C D 40 L$, Soluble CD40 ligand; $W H O$, World Health Organization. 
TABLE E2. Spearman correlation coefficient $(\rho)$ between preoperative SCD40L value and perioperative parameters

\begin{tabular}{|c|c|c|c|c|}
\hline \multirow[b]{2}{*}{ Parameter } & \multicolumn{2}{|c|}{ Preoperative } & \multicolumn{2}{|c|}{ Postoperative } \\
\hline & $\rho$ & $P$ value & $\rho$ & $P$ value \\
\hline Age (years) & -0.02 & .84 & & \\
\hline BSA $\left(\mathrm{kg} / \mathrm{m}^{2}\right)$ & 0.04 & .72 & & \\
\hline RAP (mm Hg) & 0.17 & .16 & -0.10 & .36 \\
\hline mPAP (mm Hg) & 0.05 & .64 & -0.06 & .58 \\
\hline PVR (dynes.s.cm ${ }^{-5}$ ) & 0.10 & .32 & 0.01 & .92 \\
\hline $\mathrm{CI}\left(\mathrm{L} / \mathrm{min} / \mathrm{m}^{2}\right)$ & -0.08 & .43 & -0.24 & .02 \\
\hline $\mathrm{PvO}_{2}$ (Torr) & -0.14 & .22 & 0.09 & .44 \\
\hline$\% \Delta \mathrm{PVR}$ & & & 0.03 & .76 \\
\hline
\end{tabular}

$B S A$, Body surface area; $R A P$, right atrial pressure; $m P A P$, mean pulmonary arterial pressure; $P V R$, pulmonary vascular resistance; $C I$, cardiac index; $P v O_{2}$, mixed venous oxygen pressure.

TABLE E3. Univariate logistic analysis of preoperative parameters for the risk of poor postoperative outcome subgroup

\begin{tabular}{|c|c|c|c|c|c|c|}
\hline \multirow[b]{2}{*}{ Preoperative parameter } & \multicolumn{2}{|c|}{$\begin{array}{c}\text { Post-CI subgroup } \\
\text { (good/poor, } n=74 / 16)\end{array}$} & \multicolumn{2}{|c|}{$\begin{array}{l}\text { Post-PVR subgroup } \\
\text { (good/poor, } n=77 / 13 \text { ) }\end{array}$} & \multicolumn{2}{|c|}{$\begin{array}{c}\text { Surgical outcome } \\
\text { (good/poor, } n=61 / 29)\end{array}$} \\
\hline & OR $(95 \%$ CI $)$ & $P$ & OR $(95 \%$ CI $)$ & $P$ & OR $(95 \%$ CI $)$ & $P$ \\
\hline $\mathrm{sCD} 40 \mathrm{~L}(\mathrm{ng} / \mathrm{mL})$ & $1.43(1.2-1.8)$ & .004 & $1.53(1.2-2.0)$ & .0007 & $1.74(1.3-2.3)$ & $<.0001$ \\
\hline RAP (mm Hg) & $0.99(0.8-1.2)$ & .97 & $1.1(0.9-1.3)$ & .30 & $1.0(0.9-1.2)$ & .45 \\
\hline mPAP (mm Hg) & $0.96(0.9-1.0)$ & .23 & $1.0(1.0-1.1)$ & .38 & $0.99(0.9-1.0)$ & .7 \\
\hline PVR (dynes.s.cm ${ }^{-5}$ ) & $1.00(0.9-1.0)$ & .40 & $1.00(0.9-1.0)$ & .10 & $1.00(0.9-1.0)$ & .64 \\
\hline $\mathrm{CI}\left(\mathrm{L} / \mathrm{min} / \mathrm{m}^{2}\right)$ & $0.61(0.2-1.7)$ & .34 & $0.61(0.2-1.7)$ & .34 & $0.71(0.3-1.5)$ & .37 \\
\hline PAWP (mm Hg) & $1.17(1.0-1.4)$ & .08 & $1.2(1.0-1.4)$ & .07 & $1.05(0.9-1.2)$ & .44 \\
\hline $\mathrm{PvO}_{2}$ (Torr) & $0.98(0.8-1.2)$ & .88 & $0.88(0.7-1.1)$ & .16 & $0.95(0.8-1.1)$ & .50 \\
\hline BNP (pg/mL) & $1.00(0.9-1.0)$ & .59 & $1.00(1.0-1.0)$ & .02 & $1.00(0.9-1.0)$ & .09 \\
\hline
\end{tabular}

$C I$, Cardiac index; $P V R$, pulmonary vascular resistance; $O R$, odds ratio; $C I$, confidence interval; $s C D 4 O L$, soluble CD40 ligand; $R A P$, right atrial pressure; $m P A P$, mean pulmonary arterial pressure; $P A W P$, pulmonary artery wedge pressure; $\mathrm{PvO}_{2}$, mixed venous oxygen pressure; $B N P$, brain natriuretic peptide. 
TABLE E4. Importance of Variables Based on the Decrease in Gini Impurity during 5-Fold Cross-Validation

\begin{tabular}{|c|c|c|c|c|c|c|c|c|c|c|}
\hline Ranking & First & $\begin{array}{c}\text { Mean } \\
\text { decrease } \\
\text { in Gini }\end{array}$ & Second & $\begin{array}{c}\text { Mean } \\
\text { decrease } \\
\text { in Gini }\end{array}$ & Third & $\begin{array}{c}\text { Mean } \\
\text { decrease } \\
\text { in Gini }\end{array}$ & Fourth & $\begin{array}{c}\text { Mean } \\
\text { decrease } \\
\text { in Gini }\end{array}$ & Fifth & $\begin{array}{c}\text { Mean } \\
\text { decrease } \\
\text { in Gini }\end{array}$ \\
\hline 1 & CD40L & 5.5634129 & CD40L & 5.6893019 & CD40L & 6.0929481 & CD40L & 5.6629573 & CD40L & 6.4983196 \\
\hline 2 & preCI & 2.8920639 & BNP & 3.4046506 & BNP & 2.3343697 & onset-surgery & 2.4598758 & Ddimer & 2.6608807 \\
\hline 3 & BNP & 2.3448453 & preCI & 2.3649569 & plt & 2.327759 & preCI & 2.339282 & preCI & 2.5982084 \\
\hline 4 & Ddimer & 2.0627244 & Age & 1.7915337 & onset-surgery & 2.1084613 & BNP & 2.1356553 & Age & 2.1086949 \\
\hline 5 & plt & 2.0246821 & plt & 1.6712614 & Ddimer & 1.9897174 & plt & 2.1212571 & BNP & 1.9502333 \\
\hline 6 & onset-surgery & 1.9711061 & prePAR & 1.6680089 & preCI & 1.9086334 & presPpa & 1.7645039 & plt & 1.9005514 \\
\hline 7 & predPpa & 1.8123653 & onset-surgery & 1.6399785 & SurfaceArea & 1.76179 & Ddimer & 1.7040772 & onset-surgery & 1.8179016 \\
\hline 8 & SurfaceArea & 1.6970302 & Ddimer & 1.6399733 & prePAR & 1.5678234 & prePAR & 1.6892748 & prePAR & 1.7875858 \\
\hline 9 & Age & 1.645092 & SurfaceArea & 1.54618 & Age & 1.5623541 & Age & 1.6347417 & SurfaceArea & 1.7185652 \\
\hline 10 & presPpa & 1.6164866 & presPpa & 1.5449732 & predPpa & 1.516684 & SurfaceArea & 1.5523252 & presPpa & 1.6378334 \\
\hline 11 & prePAR & 1.5870594 & predPpa & 1.4521093 & presPpa & 1.4077508 & predPpa & 1.4750563 & predPpa & 1.2971009 \\
\hline 12 & CRP & 1.5463647 & premPpa & 1.3076365 & prePra & 1.2002376 & premPpa & 1.4009785 & premPpa & 1.239195 \\
\hline 13 & prePCWP & 1.4068309 & prePCWP & 1.2524396 & premPpa & 1.1819157 & prePCWP & 1.0714686 & CRP & 1.2168087 \\
\hline 14 & premPpa & 1.3181452 & CRP & 1.0996501 & CRP & 1.0044099 & prePra & 0.9488121 & prePra & 0.9549063 \\
\hline 15 & prePra & 1.0960604 & prePra & 0.9513148 & prePCWP & 0.9372753 & CRP & 0.8402961 & prePCWP & 0.8740652 \\
\hline 16 & NYHA & 0.6392117 & NYHA & 0.5427895 & NYHA & 0.4703832 & NYHA & 0.8054876 & NYHA & 0.8480457 \\
\hline 17 & Jamieson & 0.5643612 & Jamieson & 0.3880836 & Jamieson & 0.3603922 & Jamieson & 0.4552619 & Sex & 0.4750073 \\
\hline 18 & Sex & 0.3233244 & Sex & 0.3028248 & Sex & 0.3038272 & Sex & 0.3271818 & Jamieson & 0.3962633 \\
\hline
\end{tabular}

CD4OL, Cluster of differentiation 40 ligand; preCI, Preoperative Cardiac index; BNP, Brain natriuretic peptide; plt, Platelet; Onset-surgery, Time from onset to surgery; predPpa; Preoperative diastolic pulmonary artery pressure; Surface area, Body surface area; presPpa, Preoperative systolic pulmonary artery pressure; prePAR, Preoperative pulmonary aretery resistance; $C R P, \mathrm{C}$-reactive protein; prePCWP, Preoperative pulmonary capillary wedge pressure; premPpa, Preoperative mean pulmonary aretery pressure; prePra, Preoperative right atrial pressure; NYHA, New York Heart Association functional classification; Jamieson, Jamieson classification.

TABLE E5. Comparison of intraoperative parameters, catecholamine, and postoperative ECMO support between patients with high sCD40L and those with low SCD40L

\begin{tabular}{lccc}
\hline \multicolumn{1}{c}{ Parameter } & sCD40L $<\mathbf{1 . 2 1} \mathbf{~ n g} / \mathbf{m L}$ & sCD40L $\geq \mathbf{1 . 2 1} \mathbf{~ n g} / \mathbf{m L}$ & $\boldsymbol{P}$ value \\
\hline Cardiopulmonary bypass time, min, mean \pm SD & $347 \pm 57$ & $378 \pm 110$ & .30 \\
Circulation arrest time, min, mean \pm SD & $57.5 \pm 19.4$ & $51.3 \pm 16.1$ & .32 \\
Surgery time, min, mean \pm SD & $567 \pm 131$ & $576 \pm 186$ & .89 \\
Aortic cross clamping time, min, mean \pm SD & $126 \pm 47 \pm 47$ & .69 \\
Catecholamine support, d, mean \pm SD & $1.6 \pm 0.9$ & $2.6 \pm 2.0$ & .007 \\
Postoperative ECMO, yes/no, n & $3 / 42$ & $6 / 39$ & .29 \\
Weaned from ECMO successfully, yes/no, n & $3 / 0$ & $4 / 2$ \\
\hline
\end{tabular}

Median preoperative sCD40L level: $1.21 \mu \mathrm{g} / \mathrm{mL} . s C D 40 \mathrm{~L}$, Soluble CD40 ligand; ECMO, extracorporeal membrane oxygenation. 